\title{
A Temporal Millimeter Wave Propagation Model for Tunnels Using Ray Frustum Techniques and FFT
}

\author{
Choonghyen Kwon, ${ }^{1}$ Hayeon Kim, ${ }^{1}$ Haengseon Lee, ${ }^{1}$ Hyo Hyun Choi, ${ }^{2}$ \\ Woo-jin Byun, ${ }^{3}$ and Kwangseon Kim $^{3}$ \\ ${ }^{1}$ Department of Electronic Engineering, Sogang University, 1 Sinsu-dong, Mapo-gu, Seoul 121-742, Republic of Korea \\ ${ }^{2}$ Department of Computer Science, Inha Technical College, 100 Inha-ro, Nam-gu, Incheon 402-752, Republic of Korea \\ ${ }^{3}$ Radio Technology Group, Electronics and Telecommunications Research Institute, 218 Gajeong-ro, Yuseong-gu, \\ Daejeon 305-700, Republic of Korea
}

Correspondence should be addressed to Haengseon Lee; leehs95@sogang.ac.kr

Received 14 November 2013; Revised 20 February 2014; Accepted 7 March 2014; Published 7 April 2014

Academic Editor: Felipe Cátedra

Copyright (C) 2014 Choonghyen Kwon et al. This is an open access article distributed under the Creative Commons Attribution License, which permits unrestricted use, distribution, and reproduction in any medium, provided the original work is properly cited.

A temporal millimeter wave propagation model for tunnels is presented using ray frustum techniques and fast Fourier transform (FFT). To directly estimate or simulate effects of millimeter wave channel properties on the performance of communication services, time domain impulse responses of demodulated signals should be obtained, which needs rather large computation time. To mitigate the computational burden, ray frustum techniques are used to obtain frequency domain transfer function of millimeter wave propagation environment and FFT of equivalent low pass signals are used to retrieve demodulated waveforms. This approach is numerically efficient and helps to directly estimate impact of tunnel structures and surfaces roughness on the performance of millimeter wave communication services.

\section{Introduction}

With the advent of widespread use of mobile communication services, the need for accurate wireless channel models for environments has increased [1] to ensure the quality of services for both persons and objects such as vehicles manned and unmanned. In particular, the demand for railway communication systems has increased that necessitate the use of millimeter wave band with by far large bandwidths to accommodate passenger's wireless data demand [2]. The properties of millimeter wave channels have been investigated using ray optical approaches combined with the uniform theory of diffraction [3]. Unlike other full wave analysis techniques, the ray optical or ray tracing approaches limit the interactions among scatterers to those dictated by Snell's law or law of diffractions $[4,5]$. Although the number of interactions is kept minimized, the amount of computation needed to determine whether unobstructed ray paths between interacting faces of objects exist grows enormously. To increase computational efficiency further, various space division techniques and path finding algorithms have been invented and utilized [6-13]. Among those, ray frustum techniques use frustums to form regions in which reflected, transmitted, or diffracted waves can be received [13]. The frustums help to determine whether the scattered waves reach a receiver's position and to find scattering points satisfying geometrical optics principles using virtual source positions. Those techniques have been used to predict path loss, delay spread, distribution of field strength, and so on as a function of frequency.

In this paper, more direct, practical, and numerically efficient approach to characterize millimeter wave propagation channels of tunnels is proposed which gives time domain impulse responses of the demodulated baseband signals. Time domain propagation models have been given interest in the field of ultrawide band application, where impulse responses are obtained by inverse Fourier transform or convolution of time domain responses with very wide bandwidths [14]. Due to the large bandwidths, time needed to the inversion becomes very large. The impulse responses 
presented in this paper are obtained from band pass signals and are extracted from the demodulated received signals which have undergone upconversion in the transmitter and scattering at the obstacles with upconverted frequencies. Using FFT techniques and the low pass equivalent signal concept, the number of frequency samples needed to form transfer functions can be minimized and computational efficiencies improve [15]. Considering that most wireless communication services occupy finite bandwidth, baseband signals are transformed to those of low pass equivalent which are obtained using Hilbert transform and addition process. Frequency domain transfer functions from the transmitting antenna to receiving antenna are calculated and stored using ray frustum techniques for the frequencies determined by an FFT routine. Demodulated signals in time domain can be obtained from the inverse FFT of the product of the transfer function and the low pass equivalent signal. By utilizing FFT and low pass equivalent signals, frequency-to-time domain conversions are efficiently done in seconds. To investigate the properties of wireless channels in tunnels, impulse responses are extracted for various positions and structures using the presented techniques. Experimental validation is also made for two tunnel sites.

\section{Finding Ray Paths Using Ray Frustums}

Ray tracing techniques utilizing frustums can efficiently determine whether a ray hits obstacles and the scattering points on those obstacles [13]. Data structures for ray frustums contain position vectors of vertices and normal vectors of planes enclosing each frustum. In addition, the virtual source position of each frustum is stored and used to determine the scattering point satisfying law of geometrical optics. The frustums are created using the mesh triangles of the obstacles and virtual source points. Figure 1 shows a typical tunnel structure which is modeled by triangulated cells. The frustums help the scattering points to be determined by finding the intercept point of a line and the scattering face. The line starts from the virtual source and ends at the position of the receiver. After the scattering point is found, a visibility test should be performed to determine whether an unobstructed ray path exists between the scattering point and the position of the receiving antenna. To save the time needed to perform a series of visibility tests, the data structure for the ray frustum stores indices of mesh triangles which belong to or overlap its region.

Once the ray paths from the source position to the receiver position via scattering points are found, transfer function using geometrical optics field augmented by UTD solution can be obtained at each frequency.

The outgoing electric field from the transmitting antenna along the $n$th path at a distance $R_{T}^{n}$ can be represented by the transmitter voltage:

$$
\mathbf{E}_{T}^{n}=V_{T} \sqrt{\frac{\eta}{4 \pi Z_{0}} G_{T}\left(\theta_{T}^{n}, \phi_{T}^{n}\right)} \cdot \frac{\left(h_{T, v}^{n} \widehat{\theta}_{T}^{n}+h_{T, h}^{n} \widehat{\phi}_{T}^{n}\right)}{R_{T}^{n}} e^{-j k R_{T}^{n}},
$$

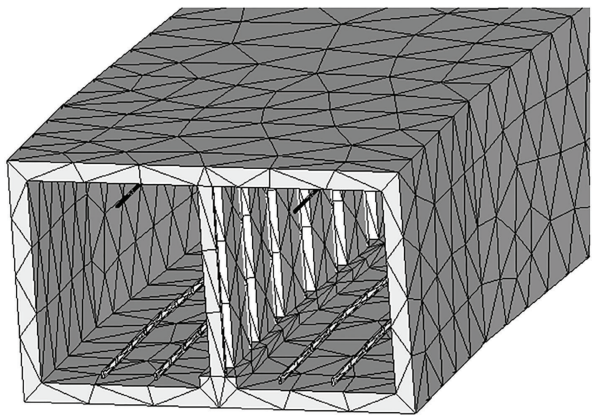

(a) Typical tunnel structure

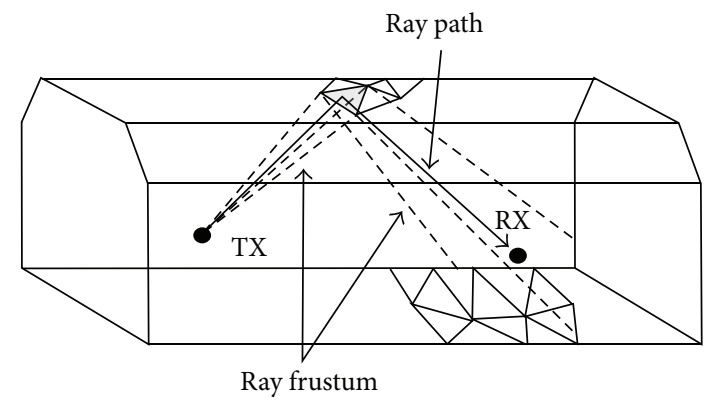

(b) Ray frustum and a ray path

FIGURE 1: Triangulated tunnel structures and ray frustums to find a ray path to $\mathrm{RX}$.

where $V_{T}$ is the transmit voltage, $\eta$ is the wave impedance of the free space, and $Z_{0}$ is the characteristic impedance of the feed line of the antenna. The gain along the $n$th ray direction $\left(\theta_{T}^{n}, \phi_{T}^{n}\right)$ is $G_{T}\left(\theta_{T}^{n}, \phi_{T}^{n}\right)$. Unit vectors $\widehat{\theta}_{T}^{n}$ and $\widehat{\phi}_{T}^{n}$ represent polarization vectors along the elevation and azimuth directions, respectively. The values $h_{T, v}^{n}$ and $h_{T, h}^{n}$ represent the polarization components [13]. Those radiated electric fields undergo reflections, diffractions, and transmissions. The voltage of the receiver is the sum of induced voltages along each path like

$$
\begin{aligned}
V_{R}= & \lambda \sqrt{\frac{Z_{0}}{4 \pi}} \sum_{n} \sqrt{G_{R}\left(\theta_{R}^{n}, \phi_{R}^{n}\right)} \\
& \cdot\left(h_{R, v}^{n} \widehat{\theta}_{R}^{n}+h_{R, h}^{n} \widehat{\phi}_{R}^{n}\right) \prod_{m} e^{-j k s_{m}^{n}} A\left(s_{m-1}^{n}, s_{m}^{n}\right) \overline{\mathbf{S}}_{m}^{n} \cdot \mathbf{E}_{T}^{n},
\end{aligned}
$$

where $A\left(s_{m-1}^{n}, s_{m}^{n}\right)$ is the divergence factor that accounts for the magnitude changes after $m$ th scattering of the $n$th path. The dyadic quantities $\overline{\mathbf{S}}_{m}^{n}$ represent reflection, transmission, or diffraction of $m$ th scattering in the $n$th path as

$$
\overline{\mathbf{S}}=\left\{\begin{array}{l}
R_{\perp} \widehat{\mathbf{e}}_{\perp} \widehat{\mathbf{e}}_{\perp}+R_{\|} \widehat{\mathbf{e}}_{\|}^{i} \widehat{\mathbf{e}}_{\|}^{r} \\
T_{\perp} \widehat{\mathbf{e}}_{\perp} \widehat{\mathbf{e}}_{\perp}+T_{\|} \widehat{\mathbf{e}}_{\|}^{i} \widehat{\mathbf{e}}_{\|}^{t} \\
-\left(\widehat{\boldsymbol{\beta}}_{0} \widehat{\boldsymbol{\beta}}_{0}^{\prime} D_{s}+\widehat{\boldsymbol{\varphi}} \widehat{\boldsymbol{\varphi}}^{\prime} D_{h}\right),
\end{array}\right.
$$

where coefficients and the unit vectors are defined in [16].

Surface roughness should be taken into account in calculating $\overline{\mathbf{S}}_{m}^{n}$ for millimeter wave frequencies as the wavelengths become comparable to the size of irregularity on 


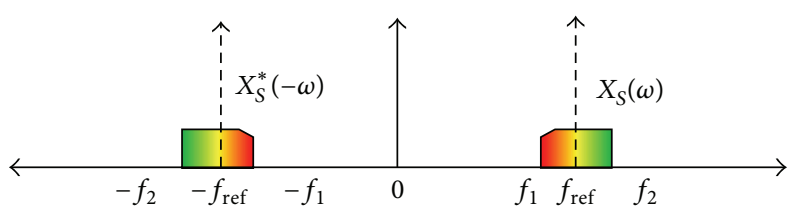

(a) Source spectrum

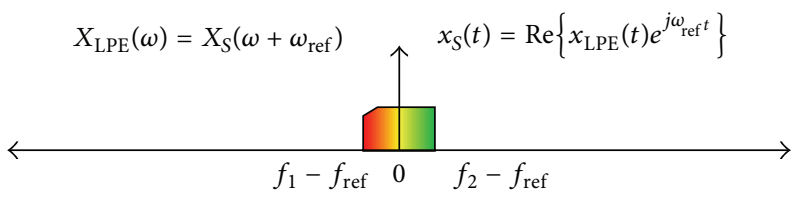

(b) Low pass equivalent signal

FIGURE 2: Low pass equivalent signal spectrum of the transmitting antenna.

obstacles [17]. A roughness factor should be multiplied on every reflection related terms. The factor is given by

$$
f\left(\theta_{i}\right)=\exp \left[-\frac{1}{2}\left(\frac{4 \pi \sigma_{s}}{\lambda} \cos \theta_{i}\right)^{2}\right],
$$

where $\sigma_{s}$ is the roughness of the surface as given by the standard deviation of the irregularities on the surface, $\lambda$ is the wavelength of the incident waves, and $\theta_{i}$ is the incident angles with respect to the normal vector of the surface.

Then, the transfer function is

$$
\begin{aligned}
H_{i}(\omega)= & \lambda \sqrt{\eta} \sum_{n=1}^{N} \sqrt{G_{R}^{n} G_{T}^{n}} \cdot\left(h_{R, v}^{n} \widehat{\theta}_{R}^{n}+h_{R, h}^{n} \widehat{\phi}_{R}^{n}\right) \\
& \cdot \prod_{m=1}^{M_{n}} e^{-j k s_{m}^{n}} A\left(s_{m-1}^{n}, s_{m}^{n}\right) \overline{\mathbf{S}}_{m}^{n} \\
& \cdot\left(h_{T, v}^{n} \widehat{\theta}_{T}^{n}+h_{T, h}^{n} \widehat{\phi}_{T}^{n}\right) \frac{e^{-j k R_{n}^{\text {inc }}}}{4 \pi R_{n}^{\text {inc }}} .
\end{aligned}
$$

To expedite calculation, frequency independent factors in the transfer function are stored in the memory and are reused. Scattering points, distances, and unit polarization vectors can be stored.

\section{Extraction of Demodulated Base Band Signal}

To retrieve time domain signal at the receiver, transfer functions at discrete frequencies should be evaluated and stored for reuse. The frequencies are fixed by the FFT of the source signal. The number of frequencies should be as small as possible to save simulation time and should be at least larger than that satisfying Nyquist sampling criterion.

Figure 2(a) shows the original source spectrum of the transmitting antenna whose spectrum has symmetry about zero frequency. Figure 2(b) is the spectrum of low pass equivalent of the original signal [15]. Because of the symmetry of the original signal, there is no loss of information after transformation. With the equivalent signal, the number of frequency samples of the transfer function from the transmitter to the receiver satisfying Nyquist criterion decreases by a factor of about $f_{\text {ref }} /\left(f_{2}-f_{1}\right)$, where $f_{\text {ref }}$ is the carrier frequency. For millimeter wave communications, the factor becomes so large that computational efficiency improves by that factor.

The number of samples of FFT to retrieve demodulated waveforms is determined by Nyquist sampling rate and the period of the signal. To simulate wireless propagation models, the period should at least be larger than maximum flight time of the wireless signals under consideration. If the period is chosen to be smaller than the flight time of the transmitted signal, intersymbol interferences or aliasings are observed. The number of sampling frequencies is determined by

$$
N=2^{\operatorname{ceil}\left[\log _{2}\left(f_{2}-f_{1}\right) T_{\max }\right]},
$$

where ceil means round up to the equal or larger nearest integer number and $T_{\max }$ is the maximum flight time of the radiated signals.

The low pass equivalent signal $X_{\mathrm{LPE}}(\omega)$ of the transmitting antenna is simply obtained by taking the FFT of the baseband signal and translating the spectrum to the carrier frequency. The voltage on the receiving antenna is

$$
Y_{\mathrm{LPE}}(\omega)=X_{\mathrm{LPE}}(\omega) H\left(\omega+\omega_{\mathrm{ref}}\right) .
$$

In calculating $H(\omega)$, surface impedances of materials, reflection and transmission coefficients, and propagation constants should be recalculated for each frequency.

Time domain representation of the low pass equivalent of the received signal is

$$
\begin{aligned}
y_{\mathrm{LPE}}(t) & =\operatorname{IFFT}\left[X_{\mathrm{LPE}}(\omega) H\left(\omega+\omega_{\text {ref }}\right)\right] \\
& =y_{\mathrm{LPE}}^{r}(t)+j y_{\mathrm{LPE}}^{i}(t) .
\end{aligned}
$$

The signal at the input port of the down-mixer is [15]

$$
\begin{aligned}
y_{\text {rec }}(t) & =\operatorname{Re}\left\{y_{\mathrm{LPE}}(t) e^{j \omega_{\mathrm{ref}} t}\right\} \\
& =y_{\mathrm{LPE}}^{r}(t) \cos \omega_{\mathrm{ref}} t-y_{\mathrm{LPE}}^{i}(t) \sin \omega_{\text {ref }} t .
\end{aligned}
$$

The downconverted signal at the output of the mixer is

$$
\begin{aligned}
v_{\mathrm{IF}}(t) & =\operatorname{LPF}\left\{\left[y_{\mathrm{LPE}}^{r}(t) \cos \omega_{\mathrm{ref}} t-y_{\mathrm{LPE}}^{i}(t) \sin \omega_{\mathrm{ref}} t\right] \cos \omega_{\mathrm{ref}} t\right\} \\
& =\frac{1}{2} y_{\mathrm{LPE}}^{r}(t) .
\end{aligned}
$$

The demodulated output of the received signal is simply obtained by taking the real part of (8).

\section{Numerical Results and Measurements}

To verify the effectiveness of the formulation in Section 2, a computer code in $\mathrm{C}++$ is developed which runs in Windows 7. First, a comparison with the measurement is presented to verify the ray frustum techniques and formulation in 


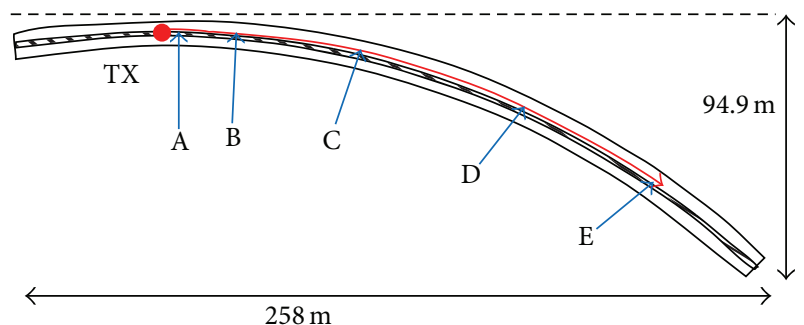

(a) Top view

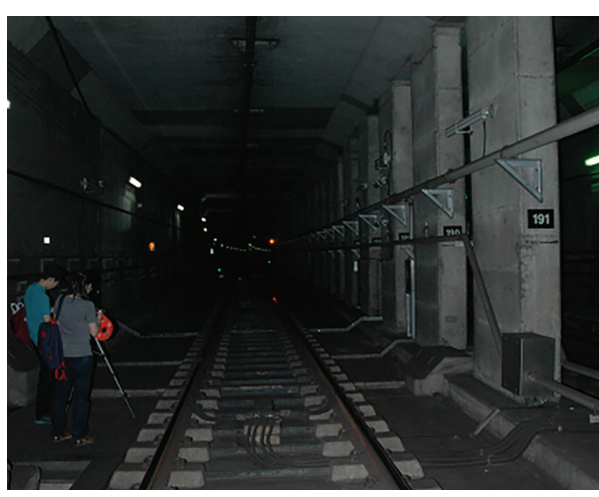

(b) Photograph of the test site

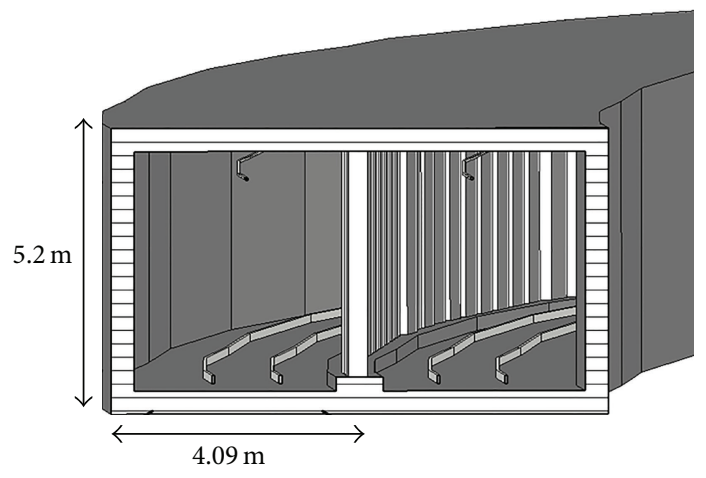

(c) Cross section

Figure 3: Dimension of a tunnel used to measure propagation loss.

the previous section. The transmitter is built with a standard gain horn antenna with a peak gain of $20 \mathrm{dBi}$ and an oscillator with an output power of $13 \mathrm{dBm}$. The transmitter radiates $24 \mathrm{GHz}$ continuous wave signal. The material constants for the interior of the tunnel are $\varepsilon_{r}=5$ and $\sigma=0.1[\mathrm{~S} / \mathrm{m}]$. The interior of the tunnel is modeled by concrete with surface roughness of $5 \mathrm{~mm}$. The test site is shown in Figure 3 which is a tunnel used for subway trains. At the center of the tunnel, concrete pillars are placed with an average spacing of $85 \mathrm{~cm}$. The average width of the pillars is $2 \mathrm{~m}$.

Figure 4 shows received power levels of measurements and simulations on the paths indicated in Figure 3, which shows good agreement. Attenuation due to cable loss of about $14 \mathrm{~dB}$ is considered and the power levels are corrected. The heights of the transmitting and receiving antennas are $1.6 \mathrm{~m}$ from the ground level of the tunnel. The directions of the antennas are parallel to those of the rails. At distances less than $13 \mathrm{~m}$ where line of sight ray paths is assured, no fluctuations of received power levels can be observed. At larger distances, multipath fading effects are shown. At the frequency of $24 \mathrm{GHz}$, surface roughness of the interior of the tunnel should be taken into account in the simulation. Without considering the diffuse scattering, power levels of the simulator are rather larger than those of measurements.

With the verified simulator, various parametric studies are performed which show the properties of the millimeter wave channel. Figure 5 shows the simulated demodulated

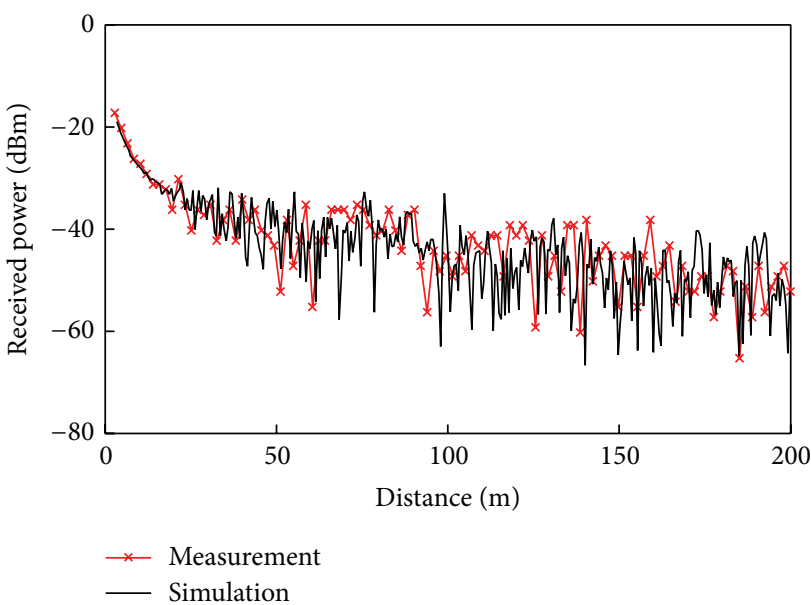

FIGURE 4: Comparison of the power levels by measurement and simulation shows good agreement.

baseband signals with the input signal in Figure 5(a) applied. The input signal is a raised cosine pulse of $10 \mathrm{~ns}$ width. The demodulated waveform is effectively an impulse response at each observation point. As the observation point becomes farther, the peak amplitude decreases and the fluctuation shows different behavior at each point. The fluctuations are due to multipath signals which undergo reflections and 


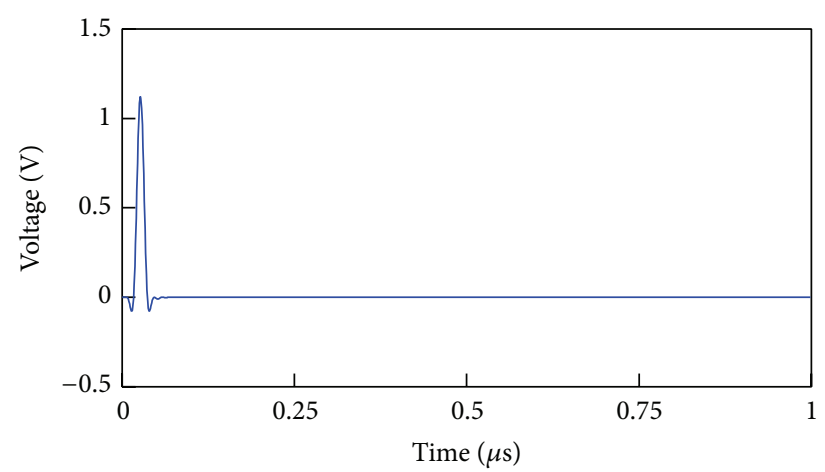

(a) Input signal

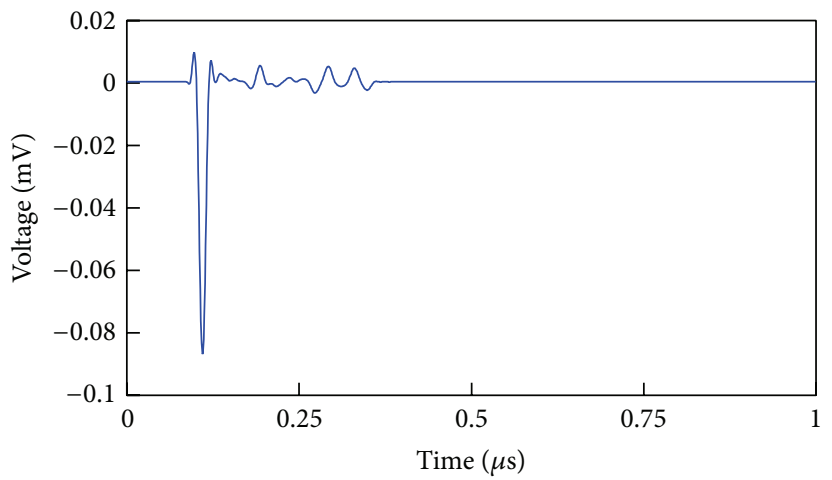

(c) At point "B"

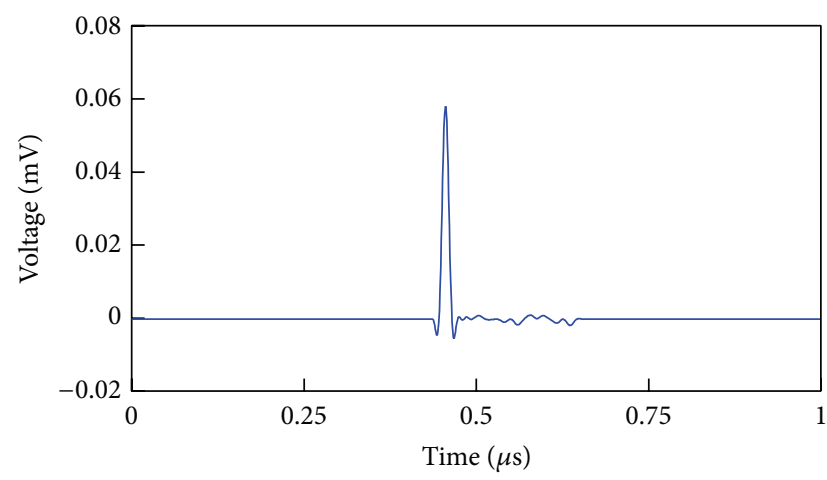

(e) At point " $D$ "

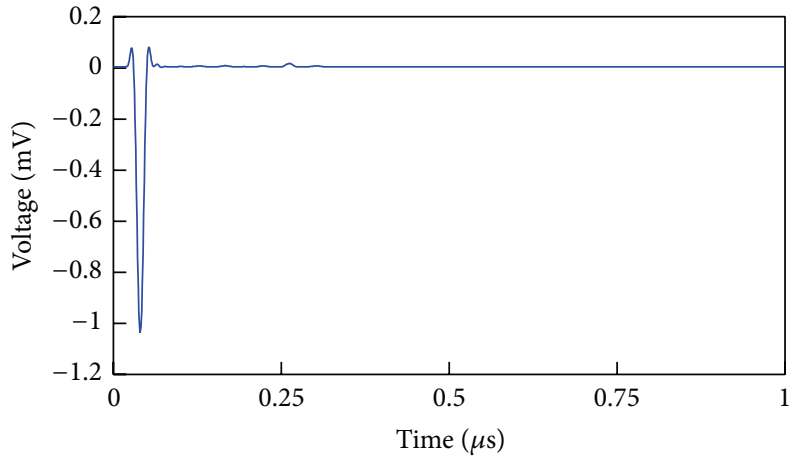

(b) At point "A"

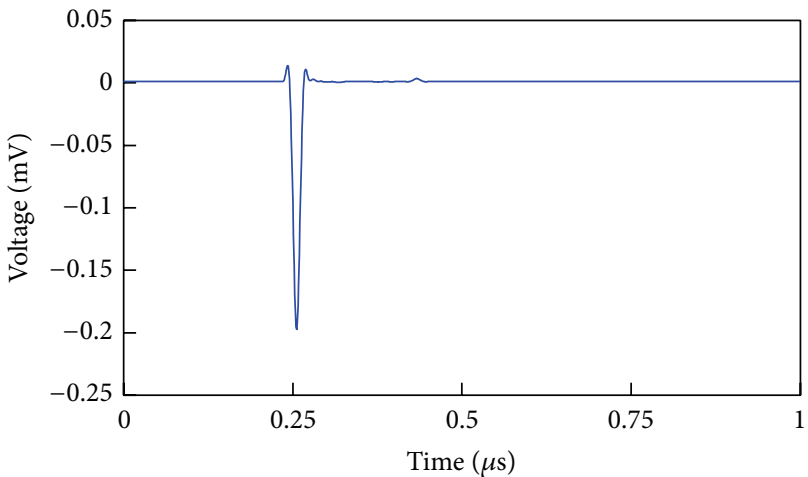

(d) At point "C"

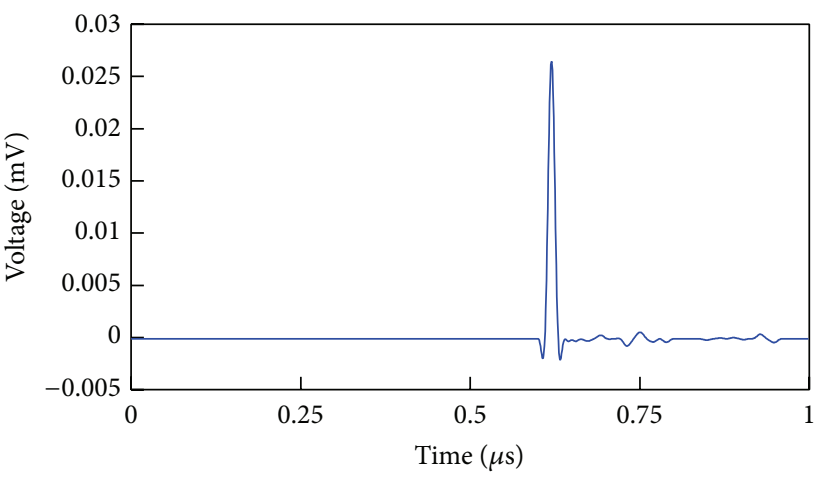

(f) At point "E"

Figure 5: Demodulated baseband signals of a curved tunnel obtained by the simulator.

diffractions on the tunnel structures. Although directional antennas are used for transmission and reception of signals and the surface roughness of the wall decreases the amplitude of reflections, multipath fading is evident due to the curvature of the tunnel.

Figure 6 shows another test site and compares the received power levels of measurements and simulations, which also shows good agreement. Metallic ornaments are attached to the interior of the tunnel and are modeled by a roughness of $2 \mathrm{~cm}$. The antennas and the oscillator used are the same as those of the previous test except that the heights of antennas are $1.1 \mathrm{~m}$. Within a distance of $15 \mathrm{~m}$, multipath scattering effect is scarce. At larger distances, fluctuation of receiving power levels is observed. If the roughness is not considered, calculated power levels are larger than measurement results at ranges farther than $50 \mathrm{~m}$. The ornaments on the wall cause diffuse scattering and increase path losses.

Figure 7 shows demodulated baseband waveforms with the same input signal in Figure 5(a) applied. Dipole antennas are used for transmitting and receiving antennas and an oscillator of $24 \mathrm{GHz}$ and $13 \mathrm{dBm}$ output power level is used for simulations. The heights of the antennas are $1.1 \mathrm{~m}$. Figures $7(\mathrm{~b})$ and $7(\mathrm{c})$ are the simulated waveforms when the interior of the tunnel is assumed to be smooth; in other words, the roughness is zero. Figures $7(\mathrm{~d})$ and $7(\mathrm{e})$ are for the case of the surface roughness equal to $2 \mathrm{~cm}$. The surface roughness causes increased attenuation of scattered waves and multipath 

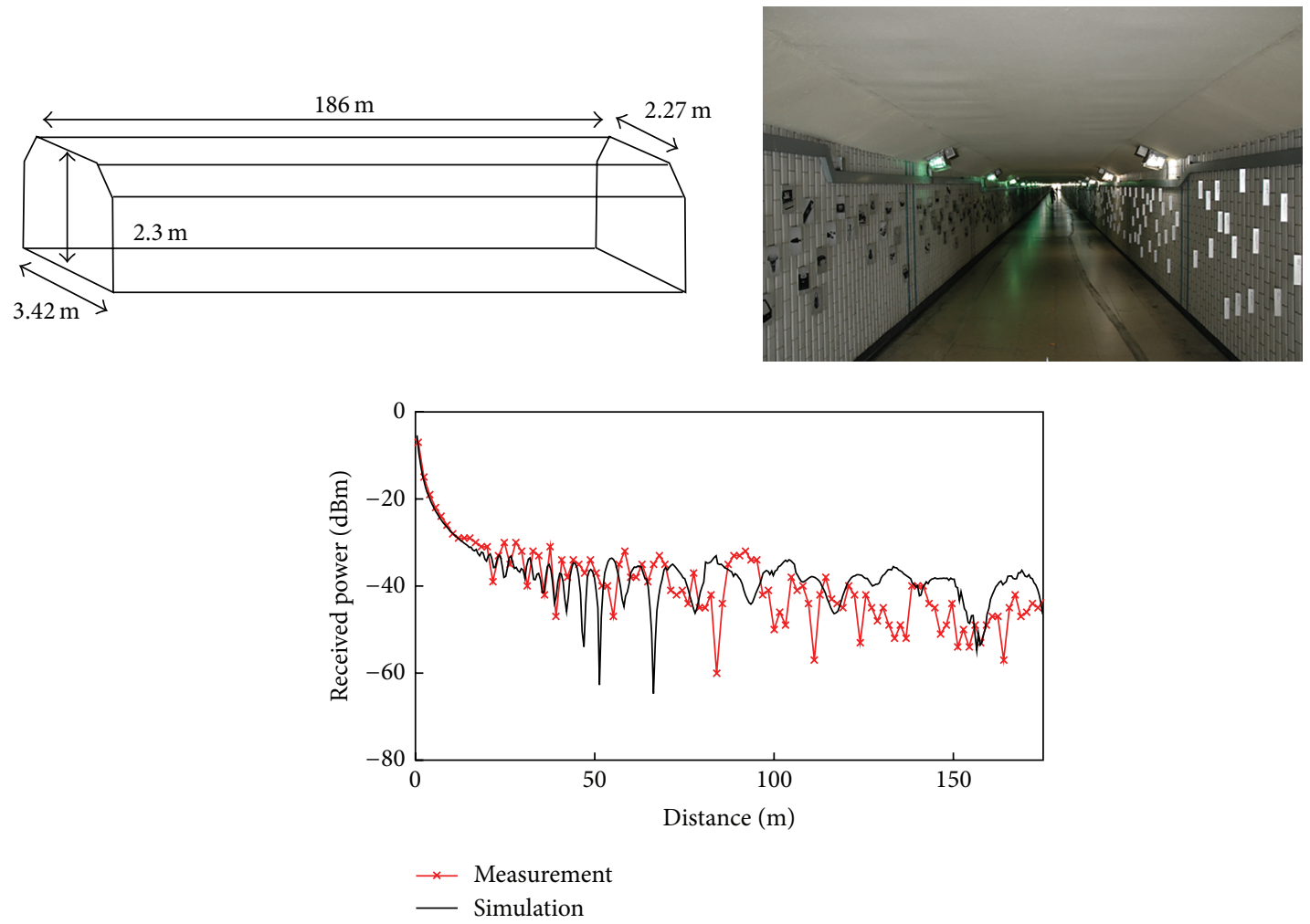

FIGURE 6: Received power levels in the second test tunnel.

effects are mitigated. Although multipath fading becomes smaller, dominant signals from the source decrease if the signals do not follow line of sight paths.

Figure 8 shows the simulated variation of power levels with the carrier frequency changed for the same site. For this simulation also, dipole antennas are used with the same directivity to compare the received power level changes. Output power levels are $13 \mathrm{dBm}$ and the height of antennas are $1.1 \mathrm{~m}$. Due to the omnidirectional radiation patterns of dipole antennas, contributions of multipath signals increase the fluctuations. Figure 8(a) shows the power levels for the smooth wall with the roughness equal to 0 . Figure $8(\mathrm{~b})$ is the case with the roughness $2 \mathrm{~cm}$. For smooth walls, the power levels are rapidly varying as the frequency increases. For a tunnel with rough surface, variations of fluctuations with frequencies are not so severe. The range of line of sight region where multipath signals have slight influence seems to increase with frequency as diffuse scattering depends on the ratio of the roughness to wavelengths. At lower frequency, the surface can be considered as a smooth surface as the wavelength is longer. The roughness of the tunnel mitigates multipath fading in a tunnel at millimeter wave frequencies.

\section{Computational Efficiency}

Figure 9 shows a series of processes to obtain time domain received signal using ray frustums and IFFT. After the triangulated mesh data of tunnel structures are loaded, they are partitioned into spatial divisions such as $\mathrm{kD}$-tree to decrease the number of unnecessary visibility tests. Then, information on transmitting antennas is loaded. Starting from the locations of transmitting antennas, ray frustums are constructed consecutively, which consumes much time (block "A" in Figure 9). The frustums are used to determine ray paths and calculate received signals. If the position of a receiver is included in a ray frustum, it means that a ray path from the transmitter to the receiver exists. The frustums are stored in a computer memory and are also partitioned into spatial divisions to minimize the number of inclusion tests. Ray frustums including the receiver only are utilized to determine the ray paths to the receiver (block " $\mathrm{B}$ " in Figure 9). Following the paths, received electric fields are calculated using geometrical optics and uniform theory of diffraction. To retrieve time domain signals at the position of the receiver, electric fields are calculated at discrete frequency spectra (block "C"). To show the computational efficiency of the proposed method, we compare the computation time spent to complete three blocks.

The tunnel structure in Figure 6 is utilized, which is triangulated into different number of cells with coarse or fine sized triangles. The frustums are generated such that the number of reflections or transmissions is set to be three, and diffractions are considered on each scattering. A PC with an Intel i7 CPU $(3.5 \mathrm{GHz})$ with $16 \mathrm{~GB}$ memory is used. To accelerate computations, a parallel processing library (OPEN-MP) is used. Computation times for the three stages are compared in Table 1. 


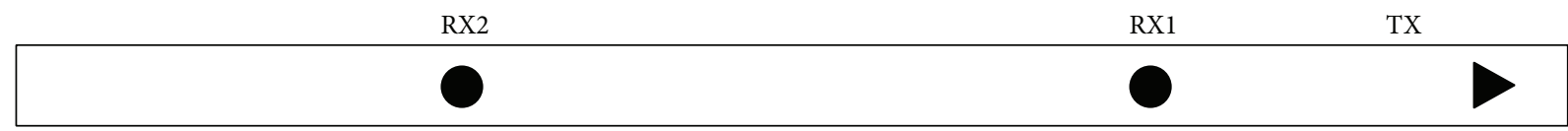

(a) Positions of the transmitter and the receivers in the tunnel

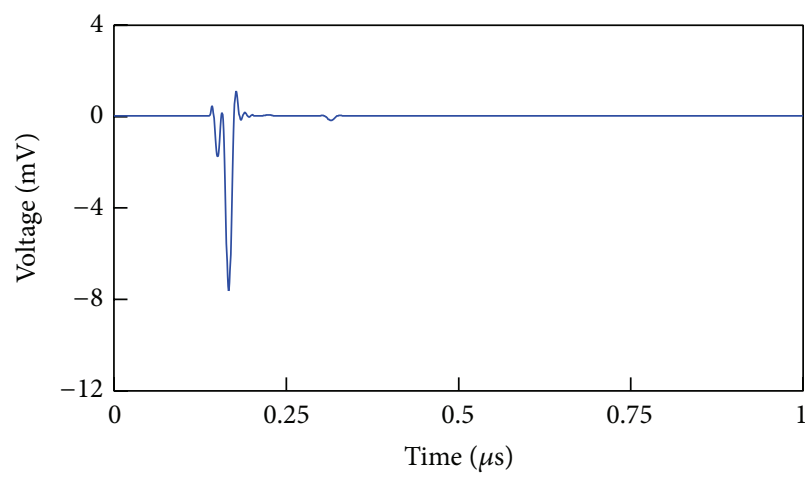

(b) At point "RX1"

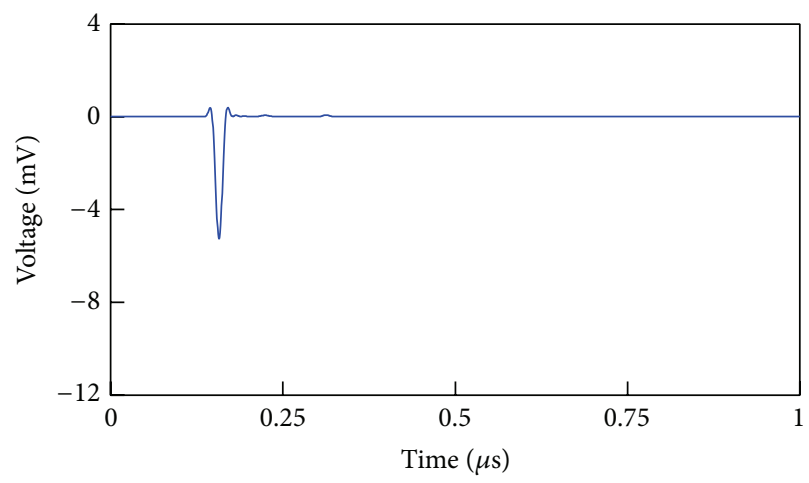

(d) At point "RX1"

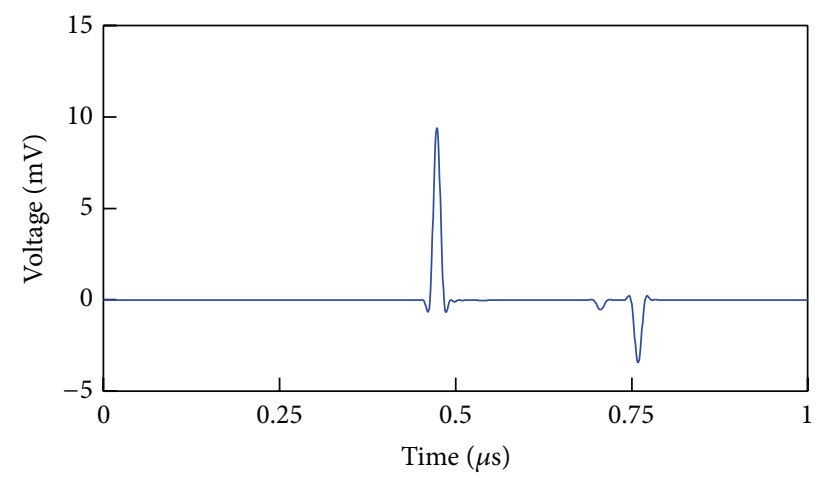

(c) At point "RX2"

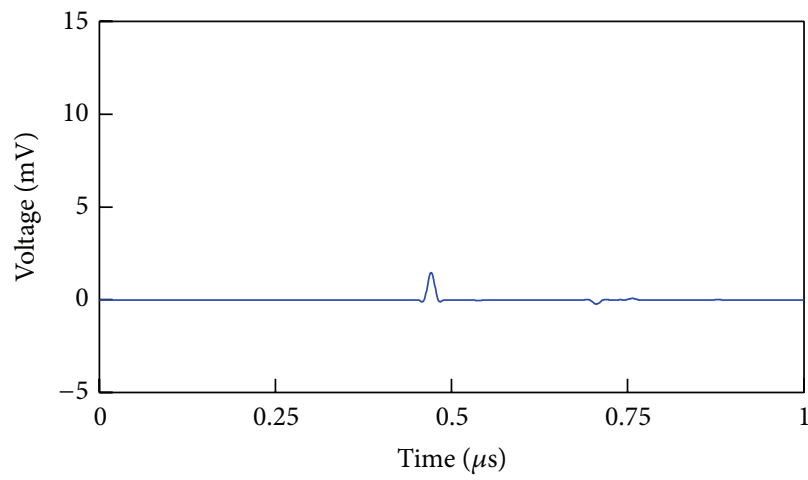

(e) At point "RX2"

FIGURE 7: Received power levels in the second test tunnel.

TABLE 1: Comparison of computation times for the tunnel structure with different level of discretization.

\begin{tabular}{|c|c|c|c|c|}
\hline Number of mesh cells & Number of frustums & Block "A" & $\begin{array}{c}\text { Block "B" } \\
(10,000 \text { points })\end{array}$ & $\begin{array}{l}\text { Block “C” (1 point) } \\
512 \text { samples, IFFT }\end{array}$ \\
\hline 1,068 & 138,550 & $14.59(\mathrm{sec})$ & $47.03(\mathrm{sec})$ & $0.47(\mathrm{sec})$ \\
\hline 3,452 & 346,900 & $39.14(\mathrm{sec})$ & $137.63(\mathrm{sec})$ & $0.47(\mathrm{sec})$ \\
\hline 5,674 & 490,241 & $72.90(\mathrm{sec})$ & $175.78(\mathrm{sec})$ & $0.485(\mathrm{sec})$ \\
\hline 9,068 & 824,330 & $146.95(\mathrm{sec})$ & $211.59(\mathrm{sec})$ & $0.53(\mathrm{sec})$ \\
\hline
\end{tabular}

Although spatial division algorithms like $\mathrm{kD}$ tree and octree are used, the computation times for the block "A" increase almost linearly with the number of mesh cells. This is because the bases of frustums are created from the mesh cells. For the block "B", computation times needed to calculate received signals at 10,000 points are compared. The computation times increase logarithmically with mesh cells. For the block " $\mathrm{C}$ ", the computation times needed to obtain time domain received signal at one observation point remain almost the same. Although the total numbers of ray frustums change with mesh cells, the number of ray paths to the fixed receiver point remains almost the same. The frequency domain transfer function from the transmitter to the receiver is calculated at 512 frequencies. The FFT result of the transmitted signal is multiplied with the transfer function and IFFT is used to retrieve time domain received signal.

\section{Conclusion}

In this paper, properties of millimeter wave propagation in tunnels are investigated in terms of frequency domain path losses and time domain impulse responses which are demodulated signals at the output of the down-mixer. To efficiently simulate the time domain results, FFT and the concept of low 


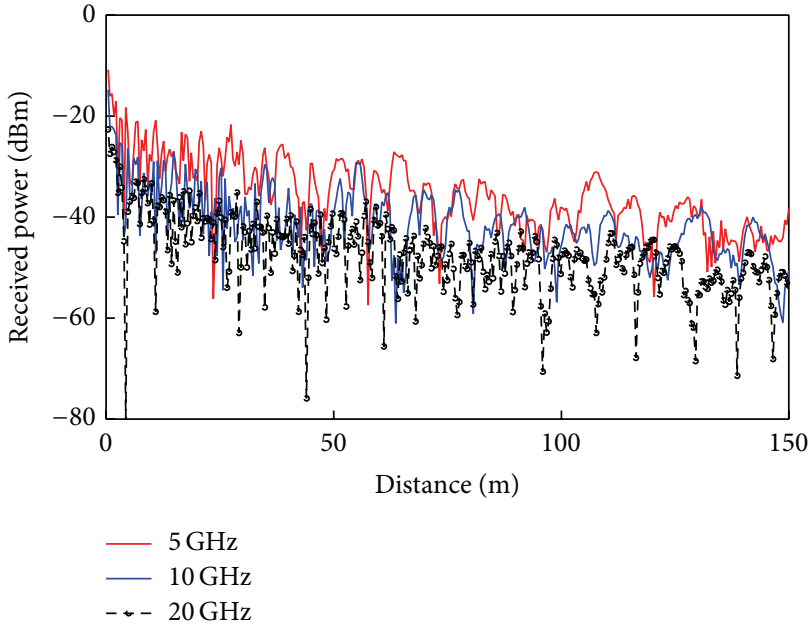

(a) Straight tunnel with roughness equal to zero

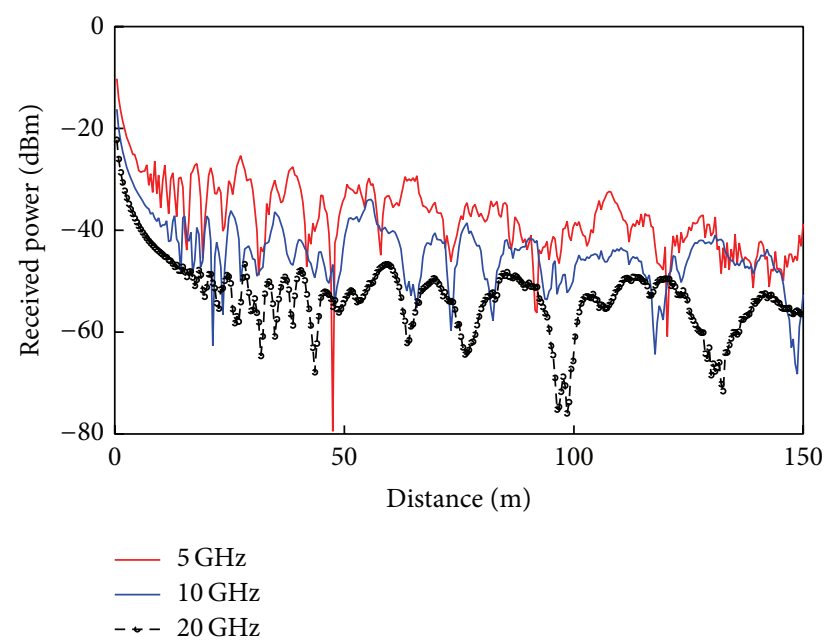

(b) A tunnel with roughness equal to $2 \mathrm{~cm}$

FIGURE 8: Received power levels with the transmitter's frequency changed.

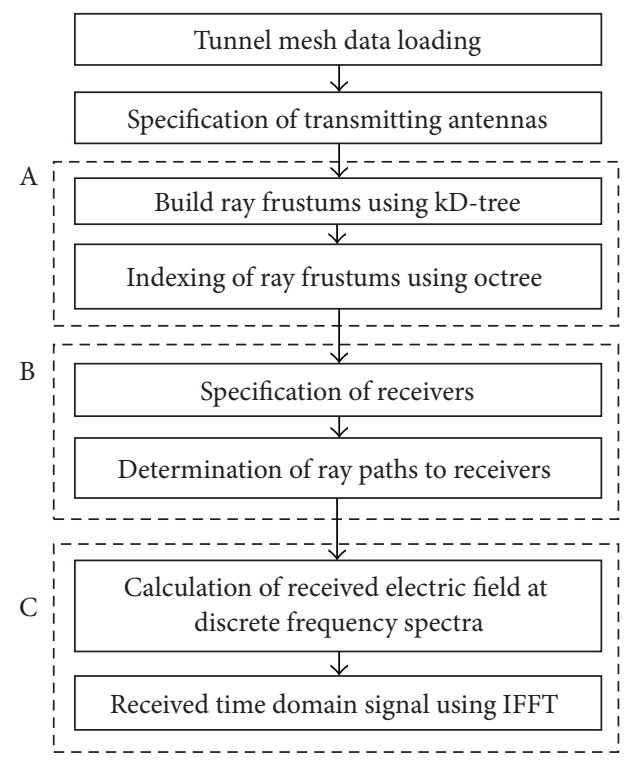

Figure 9: Flow chart to obtain time domain received signal.

pass equivalent signals are used. The impulse response shows directly the influences of scattering of waves from internal structures and surface roughness. From parametric studies using the simulator, multipath fading effects are shown to be mitigated in a tunnel with rough surface, although dominant signals strength decreases.

\section{Conflict of Interests}

The authors declare that there is no conflict of interests regarding the publication of this paper.

\section{Acknowledgments}

The authors are grateful to Dongha Kim and other members of Seoul Metropolitan Rapid Transit Corporation. This research was supported by the Basic Science Research Program through the National Research Foundation of Korea (NRF) funded by the Ministry of Science, ICT and Future Planning (no. NRF-2011-0014740).

\section{References}

[1] F. S. de Adana, O. G. Blanco, I. G. Diego, J. P. Arriaga, and M. F. Cátedra, "Propagation model based on ray tracing for the design of personal communication systems in indoor environments," IEEE Transactions on Vehicular Technology, vol. 49, no. 6, pp. 2105-2112, 2000

[2] Y. Zhou, Z. Pan, J. Hu, J. Shi, and X. Mo, "Broadband wireless communications on high speed trains," in Proceedings of the 20th Annual Wireless and Optical Communications Conference (WOCC '11), pp. 1-6, Newark, NJ, USA, April 2011.

[3] T. Kurner, D. J. Cichon, and W. Wiesbeck, "Concepts and results for $3 \mathrm{D}$ digital terrain-based wave propagation models," IEEE Journal on Selected Areas in Communications, vol. 11, no. 7, pp. 1002-1012, 1993.

[4] M. F. Cátedra, J. Pérez, F. S. de Adana, and O. Gutierrez, "Efficient ray-tracing techniques for three-dimensional analyses of propagation in mobile communications: application to picocell and microcell scenarios," IEEE Antennas and Propagation Magazine, vol. 40, no. 2, pp. 15-28, 1998.

[5] H.-W. Son and N.-H. Myung, "A deterministic ray tube method for microcellular wave propagation prediction model," IEEE Transactions on Antennas and Propagation, vol. 47, no. 8, pp. 1344-1350, 1999.

[6] Z. Yun, Z. Zhang, and M. F. Iskander, "A ray-tracing method based on the triangular grid approach and application to propagation prediction in urban environments," IEEE Transactions on Antennas and Propagation, vol. 50, no. 5, pp. 750-758, 2002. 
[7] J.-K. Bang, B.-C. Kim, S.-H. Suk, K.-S. Jin, and H.-T. Kim, “Time consumption reduction of ray tracing for RCS prediction using efficient grid division and space division algorithms," Journal of Electromagnetic Waves and Applications, vol. 21, no. 6, pp. 829840, 2007.

[8] K.-S. Jin, T.-I. Suh, S.-H. Suk, B.-C. Kim, and H.-T. Kim, "Fast ray tracing using a space-division algorithm for RCS prediction," Journal of Electromagnetic Waves and Applications, vol. 20, no. 1, pp. 119-126, 2006.

[9] Y. B. Tao, H. Lin, and H. J. Bao, "KD-tree based fast ray tracing for RCS prediction," Progress in Electromagnetics Research, vol. 81, pp. 329-341, 2008.

[10] N. S. Alvar, A. Ghorbani, and H. Amindavar, "A novel hybrid approach to ray tracing acceleration based on pre-processing \& bounding volumes," Progress in Electromagnetics Research, vol. 82, pp. 19-32, 2008.

[11] H. Suzuki and A. S. Mohan, "Frustum ray tracing technique for high spatial resolution channel characteristic map," in Proceedings of the IEEE Radio and Wireless Conference (RAWCON '98), pp. 253-256, Colorado Springs, Colo, USA, 1998.

[12] C. Lauterbach, A. Chandak, and D. Manocha, "Interactive sound rendering in complex and dynamic scenes using frustum tracing," IEEE Transactions on Visualization and Computer Graphics, vol. 13, no. 6, pp. 1672-1679, 2007.

[13] H. Kim and H. Lee, "Accelerated three dimensional ray tracing techniques using ray frustums for wireless propagation models," Progress in Electromagnetics Research, vol. 96, pp. 21-36, 2009.

[14] A. M. Attiya and A. Safaai-Jazi, "Simulation of ultra-wideband indoor propagation," Microwave and Optical Technology Letters, vol. 42, no. 2, pp. 103-108, 2004.

[15] M. C. Jeruchim, P. Balaban, and K. S. Shanmugan, Simulation of Communication Systems: Modeling, Methodology and Techniques, Springer, 2000.

[16] D. A. McNamara, C. W. I. Pistorius, and J. A. G. Malherbe, The Uniform Geometrical Theory of Diffraction, Artech House, London, UK, 1990.

[17] S. Saunders and A. Aragón-Zavala, Antennas and Propagation for Wireless Communication Systems, John Wiley \& Sons, 2007. 

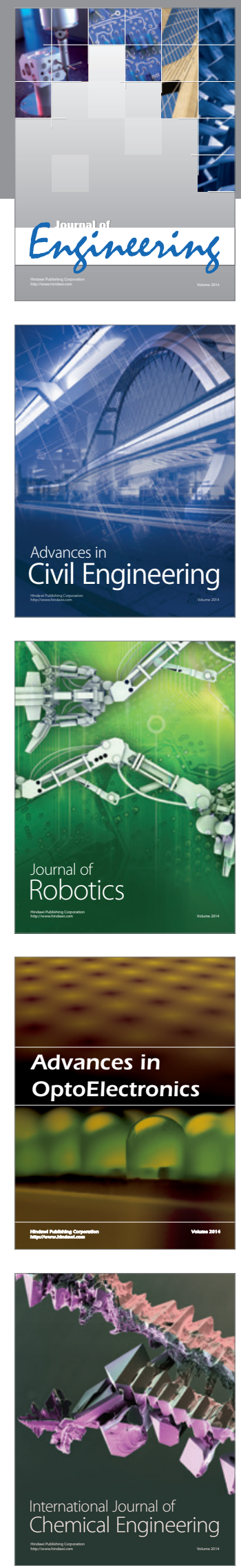

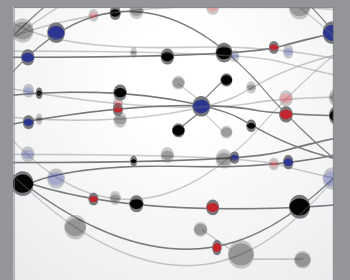

The Scientific World Journal
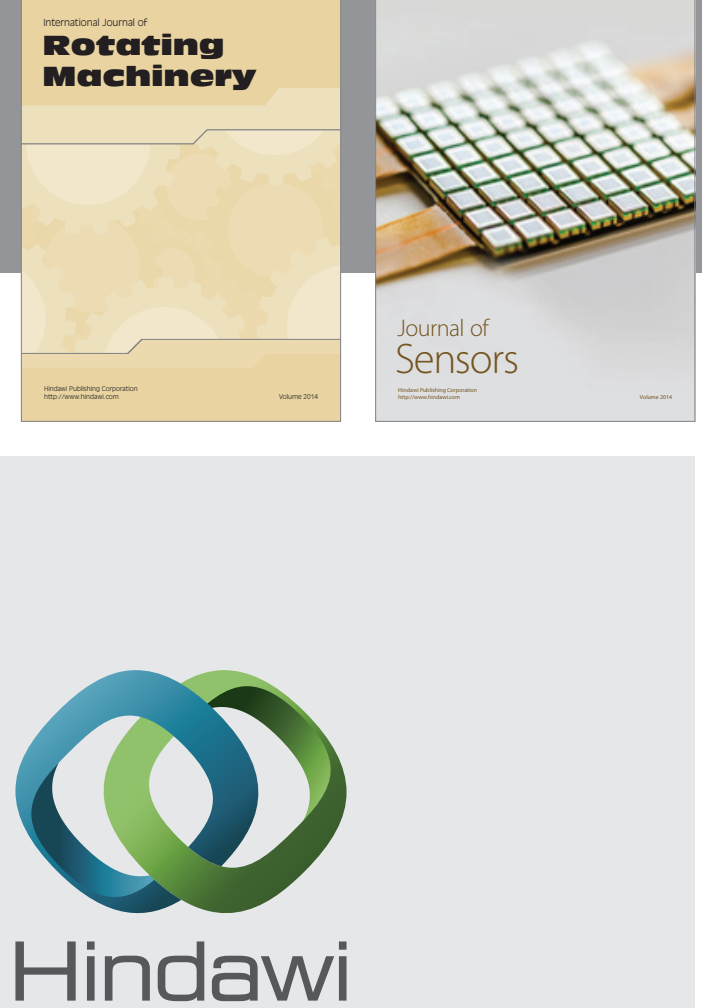

Submit your manuscripts at http://www.hindawi.com
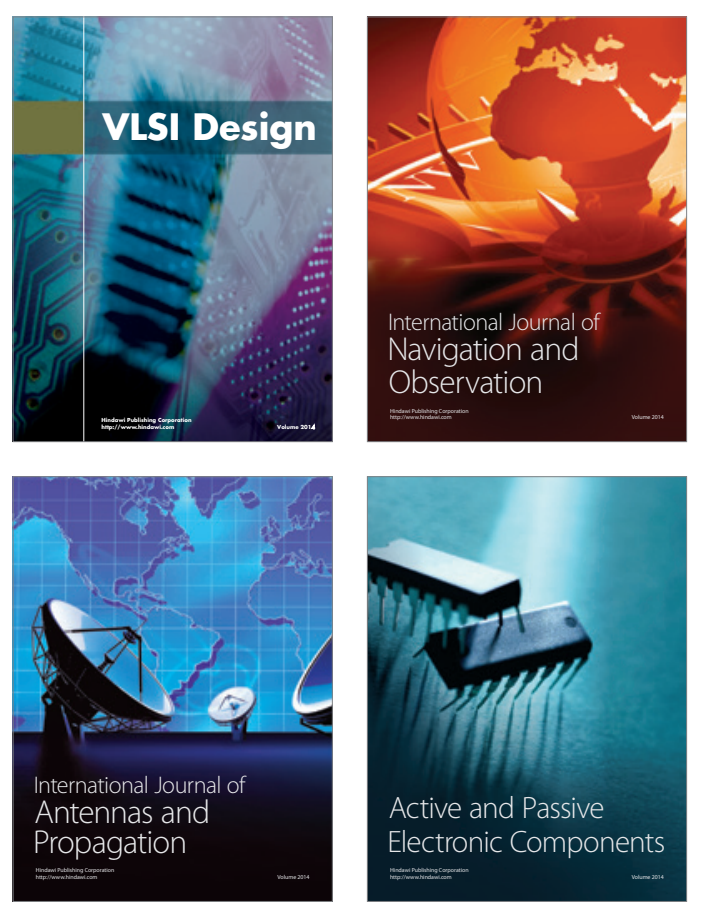
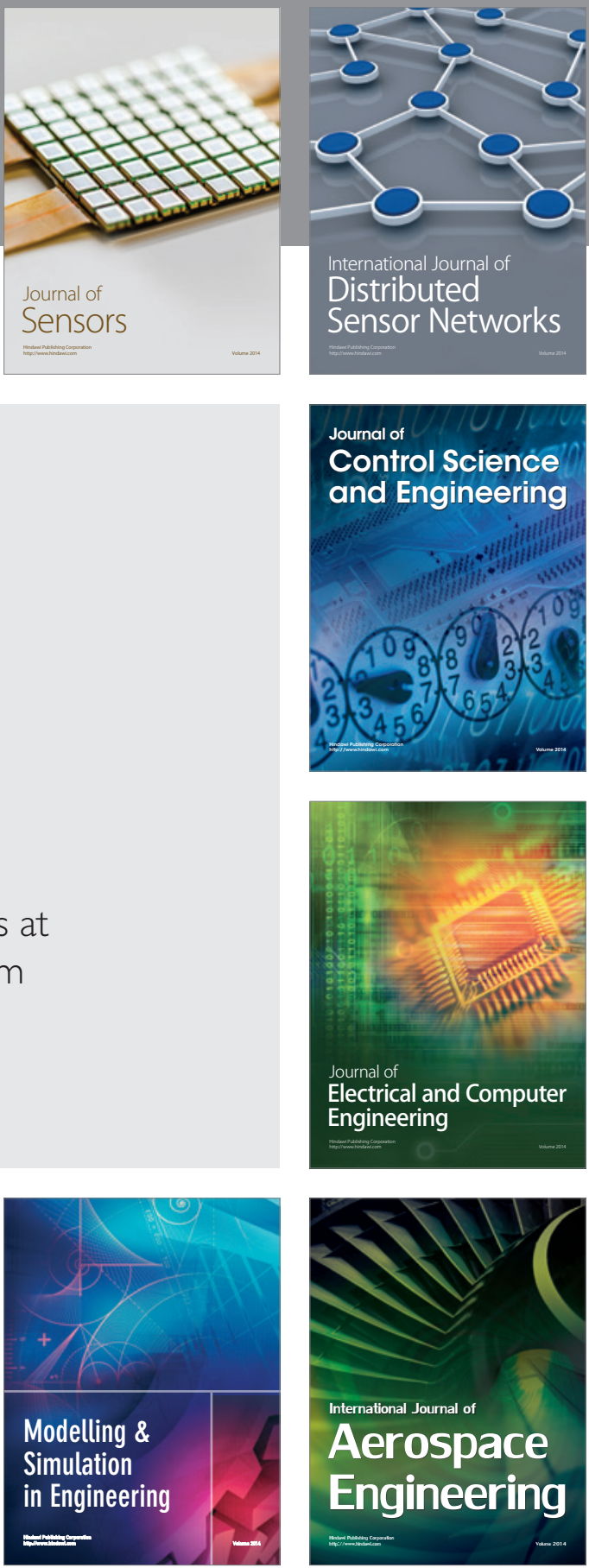

Journal of

Control Science

and Engineering
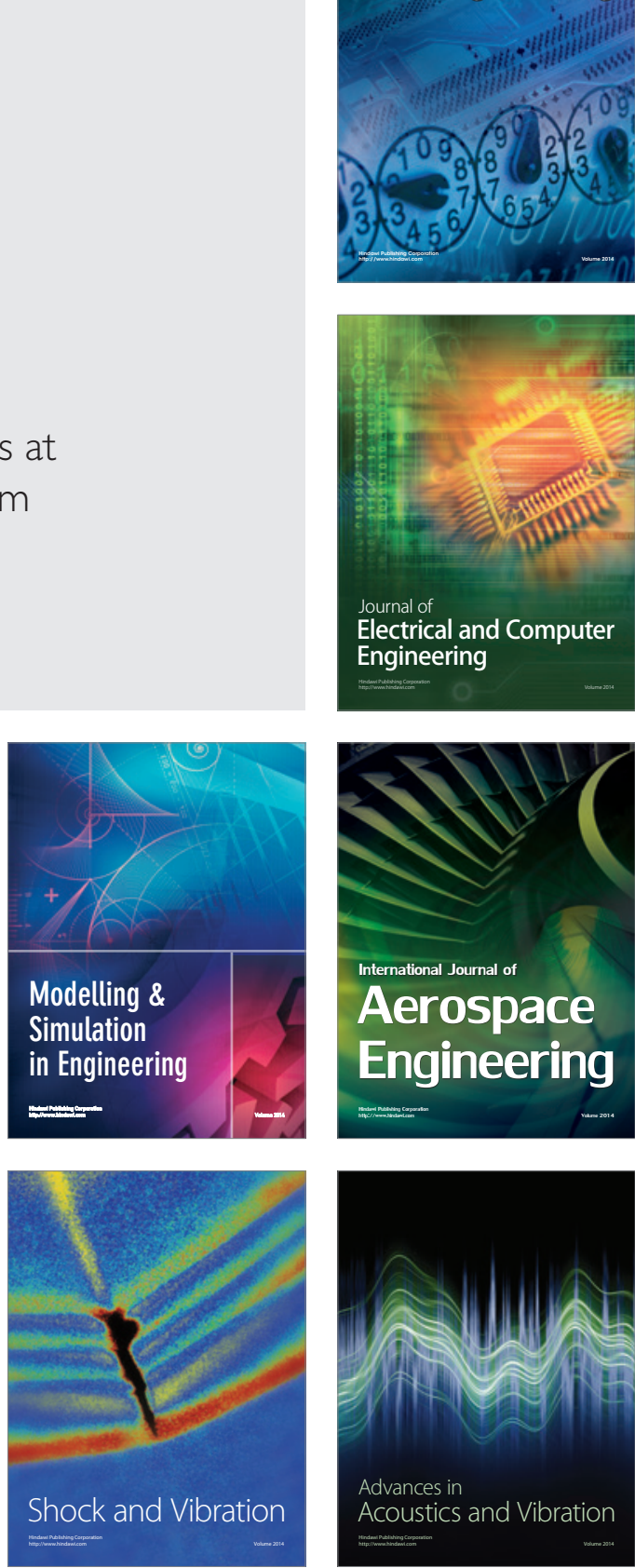